\title{
The Cannabinoid Receptor-1 Is an Imaging Biomarker of Brown Adipose Tissue
}

\author{
Olof Eriksson ${ }^{1,2}$, Kirsi Mikkola ${ }^{1}$, Daniel Espes ${ }^{3,4}$, Lauri Tuominen ${ }^{1,5}$, Kirsi Virtanen ${ }^{1}$, Sarita Forsbäck ${ }^{1}$, \\ Merja Haaparanta-Solin ${ }^{1}$, Jarmo Hietala ${ }^{1,5}$, Olof Solin ${ }^{1,6}$, and Pirjo Nuutila ${ }^{1,7}$ \\ ${ }^{I}$ Turku PET Centre, University of Turku, Turku, Finland; ${ }^{2}$ Department of Biosciences, Abo Akademi University, Turku, Finland; \\ ${ }^{3}$ Department of Medical Cell Biology, Uppsala University, Uppsala, Sweden; ${ }^{4}$ Department of Medical Sciences, Uppsala University, \\ Uppsala, Sweden; ${ }^{5}$ Department of Psychiatry, University of Turku, Turku, Finland; ${ }^{6}$ Accelerator Laboratory, Ảbo Akademi University, \\ Turku, Finland; and ${ }^{7}$ Department of Endocrinology, Turku University Hospital, Turku, Finland
}

Recently, the existence of significant deposits of brown adipose tissue (BAT) in human adults was confirmed. Its role in the human metabolism is unknown but could be substantial. Inhibition of the cannabinoid receptor-1 (CB1) by the antagonist rimonabant (SR141716) has been associated with activation of BAT thermogenesis and weight loss in mice and rats. The role of peripheral and central $C B 1$ in the activation of BAT merits further investigation. Here we developed a technique for quantifying CB1 in BAT by PET. Methods: Sections of rat BAT and subcutaneous white adipose tissue (WAT) were stained for CB1 and uncoupling protein- 1 by immunofluorescent staining. Binding of the radiolabeled CB1 antagonist (3R,5R)-5-(3-(18F-fluoromethoxy)phenyl)-3(((R)-1-phenylethyl)amino)-1-(4-(trifluoromethyl)-phenyl)pyrrolidin-2-one $\left({ }^{18} \mathrm{~F}-\mathrm{FMPEP}-\mathrm{d}_{2}\right)$ to BAT in vivo and in vitro was assessed in rats by PET. Results: We found that CB1 was colocalized with uncoupling protein-1 in BAT, but neither protein was found in WAT. Binding of the radiotracer to BAT sections (but not WAT) in vitro was high and displaceable by pretreatment with rimonabant. Deposits of BAT in rats had significant binding of ${ }^{18} \mathrm{~F}-\mathrm{FMPEP}-\mathrm{d}_{2}$ in vivo, indicating high CB1 density. WAT deposits were negative for ${ }^{18} \mathrm{~F}-\mathrm{FMPEP}-\mathrm{d}_{2}$, consistent with the immunofluorescent staining and in vitro results. Conclusion: ${ }^{18} \mathrm{~F}-\mathrm{FMPEP}-\mathrm{d}_{2}$ PET can quantify CB1 density noninvasively in vivo in rats. $C B 1$ is therefore a promising surrogate imaging biomarker for assessing the presence of BAT deposits as well as for elucidating the mechanism of CB1 antagonist-mediated weight loss.

Key Words: brown adipose tissue; cannabinoid 1 receptor; ${ }^{18} \mathrm{~F}-\mathrm{FMPEP}-\mathrm{d}_{2}$; positron emission tomography

J Nucl Med 2015; 56:1937-1941

DOI: 10.2967/jnumed.115.156422

\section{$\mathbf{R}$} ecently, the existence of significant deposits of brown adipose tissue (BAT) in human adults was confirmed (1). Its influence on the whole-body metabolism and energy expenditure in humans is currently unknown, but based on extensive experience in rodents it could be substantial (2). Quantitative noninvasive measurements of cold activated BAT glucose utilization amount to $12.2 \mu \mathrm{mol} / 100 \mathrm{~g}$

Received Feb. 24, 2015; revision accepted Aug. 4, 2015.

For correspondence or reprints contact: Olof Eriksson, Turku PET Centre, University of Turku and Turku University Hospital, P.O. Box 52, FI-20521 Turku, Finland.

E-mail: olof.eriksson@pet.medchem.uu.se

Published online Sep. 10, 2015.

COPYRIGHT (c) 2015 by the Society of Nuclear Medicine and Molecular Imaging, Inc. of BAT/min, and given that the primary energy source for BAT is fatty acids, the true energy expenditure may be higher (1). Consequently, pharmaceutical means for inducing subcutaneous white adipose tissue (WAT) to BAT conversion (browning) as well as BAT activation are pursued for the treatment of obesity.

It has been shown that chronic inhibition of the cannabinoid receptor-1 (CB1) by the antagonist rimonabant (SR141716) is associated with activation of BAT thermogenesis and weight loss in mice and rats $(3,4)$. These effects were shown to be mediated by both peripheral CB1 (5) and CB1 located in the central nervous system (CNS) (4).

Furthermore, chronic treatment by rimonabant induced an increase in uncoupling protein-1 (UCP-1) in WAT and initiated a transdifferentiation into a brown fat phenotype (6). Clearly, the role of peripheral and central $\mathrm{CB} 1$ in the activation of BAT merits further investigation.

However, the clinical translation of these interesting results has been hampered by the lack of methodologies for the noninvasive in vivo study of peripheral CB1. The PET ligand (3R,5R)-5-(3-(18Ffluoromethoxy)phenyl)-3-(((R)-1-phenylethyl)amino)-1-(4-(trifluoromethyl)-phenyl)pyrrolidin-2-one $\left({ }^{18} \mathrm{~F}-\mathrm{FMPEP}-\mathrm{d}_{2}\right)$ has previously been validated for quantification of CB1 availability and occupancy in the CNS in humans (7).

In this study, we investigated the feasibility of assessing CB1 availability in deposits of BAT using ${ }^{18}$ F-FMPEP- $\mathrm{d}_{2}$ PET.

\section{MATERIALS AND METHODS}

\section{Staining for CB1 and UCP-1 in Adipose Tissue}

BAT and subcutaneous WAT were excised from Sprague-Dawley rats after euthanasia. The tissue samples were immediately snap-frozen, mounted in Tissue-Tec (Sakura), and processed into 10- $\mu \mathrm{m}$ sections by a Microtome (Microm) and placed on object glasses.

The sections were fixed in ice-cold acetone for $10 \mathrm{~min}$ and then blocked with DAKO serum-free protein block for $60 \mathrm{~min}$ (Agilent Technologies). The sections were then incubated overnight at $4{ }^{\circ} \mathrm{C}$ with either an anti-UCP-1 antibody (Ab\#10983 [Abcam]; dilution, 1:500) or an anti-CB1 antibody (Ab\#23703 [Abcam]; dilution, 1:300) and then incubated with the secondary antibody Alexa fluor 555 (Invitrogen; donkey antirabbit; dilution, 1:1,000) for $60 \mathrm{~min}$ in a humidified dark chamber. The slides were mounted with ProLong Gold Antifade reagent with 4',6-diamidino-2-phenylindole (DAPI) (Life Technologies). Adjacent frozen sections were fixed in ethanol and stained with hematoxylin and eosin. Images were acquired with a Zeiss LSM780 confocal microscope at $20 \times$ magnification. The sections stained for UCP-1 were assigned a green pseudocolor. 


\section{Radiochemistry}

${ }^{18} \mathrm{~F}-\mathrm{FMPEP}-\mathrm{d}_{2}$ was synthesized as described previously (8). The radiochemical purity was greater than $95 \%$ and the specific radioactivity greater than $500 \mathrm{GBq} / \mu \mathrm{mol}$ at the end of synthesis.

\section{In Vitro Autoradiography}

Biopsies of intrascapular BAT (iBAT) and subcutaneous WAT from Sprague-Dawley rats were frozen to $-80^{\circ} \mathrm{C}$ and processed into $10-\mu \mathrm{m}$ sections. To study tracer binding properties to the tissue, the sections were incubated in concentrations in the nanomolar range $(0.05-1.5 \mathrm{nM})$ of ${ }^{18}{ }^{\mathrm{F}-F M P E P}-\mathrm{d}_{2}$ in $50 \mathrm{mM}$ Tris-HCl (pH 7.6) for $90 \mathrm{~min}$ at room temperature. Nondisplaceable binding was assessed by adding $10 \mu \mathrm{M}$ of the CB1 antagonist rimonabant (rimonabant hydrochloride; Sigma-Aldrich) $10 \mathrm{~min}$ before ${ }^{18} \mathrm{~F}-\mathrm{FMPEP}-\mathrm{d}_{2}$. Tissue slices were then washed 3 times for $4 \mathrm{~min}$ in $50 \mathrm{mM}$ ice-cold Tris to remove excess tracer and then air-dried. The sections were exposed against a phosphor-imager screen (Fuji BAS-TR2025; Fuji Photo Film Co.) for $3 \mathrm{~h}$, digitalized using a BAS-5000 Fuji scanner (Fujifilm Life Science), and analyzed using the AIDA 4 (Raytest) software.

\section{Organ Distribution Studies}

Sprague-Dawley rats ( $n=12$, male; age, 8-10 wk; weight, $292 \pm 24 \mathrm{~g})$ were housed under standard laboratory conditions with free access to food and water. All handling and experiments were performed in accordance with the national guidelines and were approved by the local ethics committee for animal research. All applicable institutional or national guidelines for the care and use of animals were followed.

${ }^{18} \mathrm{~F}-\mathrm{FMPEP}-\mathrm{d}_{2}(9.6 \pm 2.3 \mathrm{MBq}$, corresponding to $33.2 \pm 9.2 \mathrm{MBq} / \mathrm{kg}$ or in all cases $<0.1 \mu \mathrm{g} / \mathrm{kg}$, adhering to the tracer concept) was administered intravenously in the tail in rats sedated by isoflurane. Anesthesia was induced by $4 \%$ isoflurane in $600 \mathrm{~mL}$ air/min and maintained by $2.5 \%$ isloflurane in $400 \mathrm{~mL}$ air/min. The animals were maintained under anesthesia and sacrificed at different time points: 5, 15, 30, 60, 90, and $120 \mathrm{~min}$. Tissues were excised and measured for radioactive content in a well counter and corrected for weight, and radioactive uptake was expressed as percentage injected dose per gram of tissue.

Portions of the iBAT deposit, the CNS cortex, subcutaneous WAT, and blood plasma were taken for analysis of radioactive metabolites as described below.

\section{Metabolite Analysis}

Metabolite analysis was performed on tissues from 6 animals at 6 different time points: $5,15,30,60,90$, and $120 \mathrm{~min}$.

Samples from iBAT, cortex, and subcutaneous WAT was homogenized in $1 \%$ trifluoroacetic acid (TFA)/acetonitrile in 2-mL borosilicate tubes using a pestle. The liquid containing homogenates was transferred to 2-mL Eppendorf tubes. Blood plasma $(250 \mu \mathrm{L})$ was mixed with 350 $\mu \mathrm{L}$ of $1 \%$ TFA/acetonitrile in a 2 -mL Eppendorf tube.

All samples were centrifuged at 4,500 rpm for $4 \mathrm{~min}$, and the supernatant was transferred to a new Eppendorf tube. Additional $1 \%$ TFA/acetonitrile was added, and the centrifugation was repeated until the resulting liquid was clear.

Aliquots $(10-20 \mu \mathrm{L})$ of each tissue homogenate and a $2-\mu \mathrm{L}$ reference of ${ }^{18} \mathrm{~F}$-FMPEP- $\mathrm{d}_{2}$ in human plasma were added to a thin-layer chromatography plate. One percent TFA $(5 \mathrm{~mL})$ was added to cover the bottom centimeter of a glass vat, and the thin-layer chromatography plate was placed standing in the buffer. The plate was left for approximately $1 \mathrm{~h}$ until the buffer had reached the 5-cm mark.

The plate was then dried and exposed against a digital autoradiographic plate (Fuji BAS-TR2025; Fuji Photo Film Co.) for more than $2 \mathrm{~h}$ and then scanned in a BAS-5000 Fuji scanner (Fujifilm Life Science). The autoradiograms of the radioactive ${ }^{18} \mathrm{~F}-\mathrm{FMPEP}-\mathrm{d}_{2}$ metabolites were analyzed by AIDA 4 (Raytest). Peaks were integrated and background levels subtracted. The peak corresponding to native, unchanged ${ }^{18} \mathrm{~F}-\mathrm{FMPEP}-\mathrm{d}_{2}$ ligand was identified by comparison with the reference. The percentage of unchanged ${ }^{18}$ F-FMPEP- $\mathrm{d}_{2}$ in each sample was determined by calculating the contribution of the area of this peak compared with the total integrated area of all peaks.

The percentage of native ${ }^{18} \mathrm{~F}-\mathrm{FMPEP}-\mathrm{d}_{2}$ in all tissues over time was further analyzed in GraphPad Prism 5.04 (GraphPad Software). The metabolism in blood plasma was fitted to a monoexponential function for further use in the construction of an input function for tracer kinetic analysis.

\section{PET Imaging Procedures}

Seven of the animals were examined by a preclinical PET/CT scanner (Inveon PET/CT; Siemens). The animals were positioned by $\mathrm{CT}$, with the brain and the abdomen in the $127-\mathrm{mm}$ axial field of view.

The animals were then examined by PET in list-mode for $120 \mathrm{~min}$ from injection of ${ }^{18} \mathrm{~F}-\mathrm{FMPEP}-\mathrm{d}_{2}$.

PET data were reconstructed into the frame sequence $11 \times 10,1 \times 20$, $5 \times 30,1 \times 45,4 \times 60,1 \times 330,2 \times 600,1 \times 900$, and $4 \times 1,200$. Images were acquired in 3-dimensional mode and reconstructed using an iterative ordered-subset expectation maximization 2-dimensional algorithm.

\section{PET Analysis}

Reconstructed data were analyzed by PMOD (PMOD Technologies Ltd.). Regions of interest of select tissues were delineated on coregistered transaxial CT slices.

The left heart ventricle was used as a model for arterial tracer content and as a basis for an input function for kinetic tracer analysis. The left ventricle was delineated on early PET time frames in combination with CT images to avoid mismatch due to breathing movement artifacts. iBAT, subcutaneous WAT, and CNS cortex were delineated on timesummated PET images and corresponding coregistered CT images. Cortical bone was delineated in the skull to assess the extent of tracer defluorination also seen in the organ distribution study.

Time-activity curves (TACTs) (expressed as $\mathrm{kBq} / \mathrm{mL}$ ) in all the described tissues were then generated for the full 120-min sequence.

The individual left ventricle TACT was used as a model for whole arterial blood. The plasma-to-blood ratio was extrapolated by fitting a monoexponential function to data from the ex vivo organ distribution (5- to 120-min time points). This correction was then applied to each individual left ventricle TACT.

A monoexponential curve was similarly fitted to the data from the plasma metabolite data. This function, describing the percentage of unchanged ${ }^{18} \mathrm{~F}-\mathrm{FMPEP}-\mathrm{d}_{2}$ ligand in plasma on a population level, was then applied as a final correction to generate a metabolite-corrected arterial plasma curve for use as an input function for tracer kinetic modeling.

The TACTs of iBAT, cortex, and subcutaneous WAT were used as output data in the PMOD kinetic modeling module (PKIN; PMOD Technologies). One- and 2-tissue-compartment (1TC and 2TC, respectively) models (with variable vascular contribution, $\mathrm{Vb}$, as a parameter) were applied to each tissue using the metabolitecorrected arterial plasma curve as input, and the uncorrected individual left ventricle TACT was used as a model for arterial whole-blood and vascular contribution. Tissue uptake of tracer was expressed as total volume of distribution $(\mathrm{Vt})$ or volume of distribution of the second compartment (Vs, interpreted as receptor specific binding).

\section{RESULTS}

\section{CB1 in Adipose Tissue}

Sections of iBAT were positive for immunostaining with CB1 (Fig. 1A) as well as UCP-1 (Fig. 1C). Predictably, UCP-1 staining was negative in WAT sections (Fig. 1D). WAT samples were also negative for CB1 immunostaining (Fig. 1B). Hematoxylin and eosin staining of adjacent sections confirmed the distinct morphology of BAT (Fig. 1E) and WAT (Fig. 1F). 


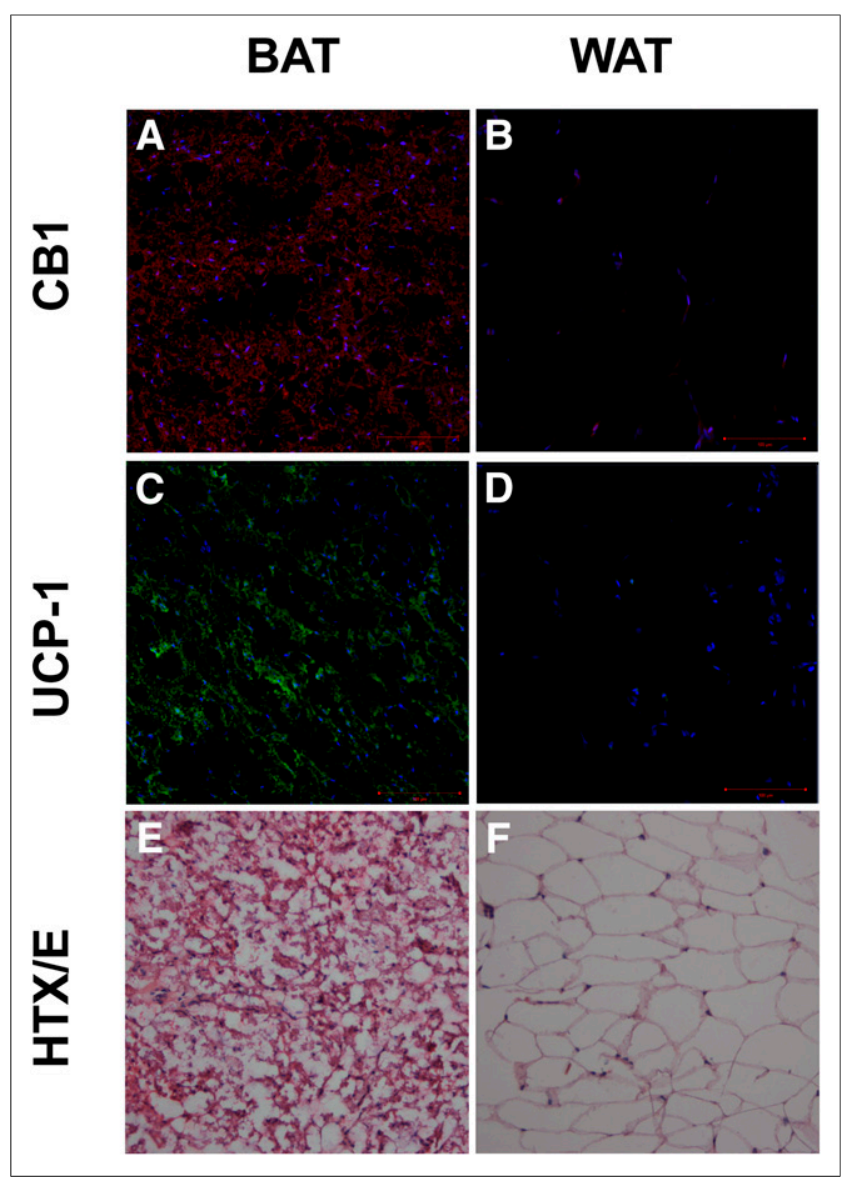

FIGURE 1. Immunohistochemistry of $\mathrm{CB} 1$ and UCP-1 in iBAT and WAT. BAT was positive for CB1 (A) and for UCP-1 (C). WAT, on the other hand, was negative both for CB1 (B) and for UCP-1 (D). Hematoxylin and eosin (HTX) staining of adjacent sections confirmed distinct morphology of BAT (E) and WAT (F). Scale bar indicates $100 \mu \mathrm{m}$.

\section{In Vitro Autoradiography}

Displaceable, or CB1-mediated, binding was approximately 10 -fold higher in sections of BAT than WAT $\left(P<0.01 ;{ }^{18} \mathrm{~F}-\mathrm{FMPEP}-\mathrm{d}_{2}\right.$ concentration, $0.5-1.5 \mathrm{nM}$; Fig. 2A). ${ }^{18} \mathrm{~F}-\mathrm{FMPEP}-\mathrm{d}_{2}$ binding in iBAT, but not WAT, could be blocked by coincubation with the CB1 antagonist rimonabant. Saturation of binding occurred at nanomolar concentrations of ${ }^{18} \mathrm{~F}-\mathrm{FMPEP}-\mathrm{d}_{2}$, with a corresponding estimated receptor density of $667 \mathrm{fmol} \mathrm{CB} 1 \mathrm{~s}$ per microgram of BAT tissue (Fig. 2B). No appreciable CB1-specific binding was found in WAT at either tracer concentration used, thus neither dissociation constant nor receptor density could be estimated (Fig. 2C).

\section{PET Quantification of CB1 in Rats}

iBAT deposits were clearly identifiable on PET images (dark blue region, Fig. 3A). A high accumulation of radioactivity was also seen in cortical bone (light blue region), most likely corresponding to incorporation of ionic ${ }^{18} \mathrm{~F}^{-}$in hydroxyapatite in bone tissue. The free ionic ${ }^{18} \mathrm{~F}^{-}$was likely due to species-specific defluorination of ${ }^{18} \mathrm{~F}$-FMPEP- $\mathrm{d}_{2}$. Red regions in Figure $3 \mathrm{~B}$ indicate subcutaneous WAT. Ex vivo organ distribution confirmed marked accumulation of radioactivity in iBAT and brain including the cortex (Fig. 3C). Accumulation in subcutaneous WAT was lower than for iBAT 120 min after administration, consistent with the immunostaining $(P<0.001)$. The residual tracer accumulation in WAT, despite an almost complete lack of CB1 expression, is likely due to nonspecific binding because of the relatively high lipophilicity of ${ }^{18} \mathrm{~F}-\mathrm{FMPEP}-\mathrm{d}_{2}$.

The plasma-to-whole-blood ratio was best estimated by a monoexponential decay function (described by the parameters $\mathrm{Y}_{0}=1.56$, $\mathrm{k}=0.025$, and plateau $=1.35$ ).

Metabolite analysis showed that radioactive uptake in iBAT, cortex, and subcutaneous WAT originated almost exclusively from unchanged ${ }^{18}$ F-FMPEP- $\mathrm{d}_{2}$ for the duration of the experiment (5-120 min) (Fig. 3D). The metabolic degradation of ${ }^{18} \mathrm{~F}-\mathrm{FMPEP}-\mathrm{d}_{2}$ in blood plasma followed a monoexponential decay function (described by the parameters $\mathrm{Y}_{0}=100, \mathrm{k}=0.038$, and plateau $=37.9$ ).

A 2TC model best described tracer kinetics in iBAT and CNS cortex in all subjects, based on minimization of $\chi^{2}$ (BAT $\chi^{2}, 5.2 \pm$ 2.3; cortex $\chi^{2}, 5.0 \pm 2.2$ ). There was no difference between Vs and $\mathrm{Vt}$ between these tissues, indicating a similar amount of available receptors.

A 1TC model best described the tracer kinetics in subcutaneous WAT, whereas the 2TC model did not converge. The same 1TC model could also reasonably well describe the kinetic behavior in iBAT $\left(\chi^{2}, 7.7 \pm 2.9\right)$. A comparison between WAT and BAT was therefore performed using the 1TC model. There was a significant difference in Vt between WAT and iBAT (Fig. 3E). There was no difference in the vascular contribution. The difference in Vt was due to a higher $K_{1}$ and to a lesser extent $k_{2}$ in iBAT compared with WAT.
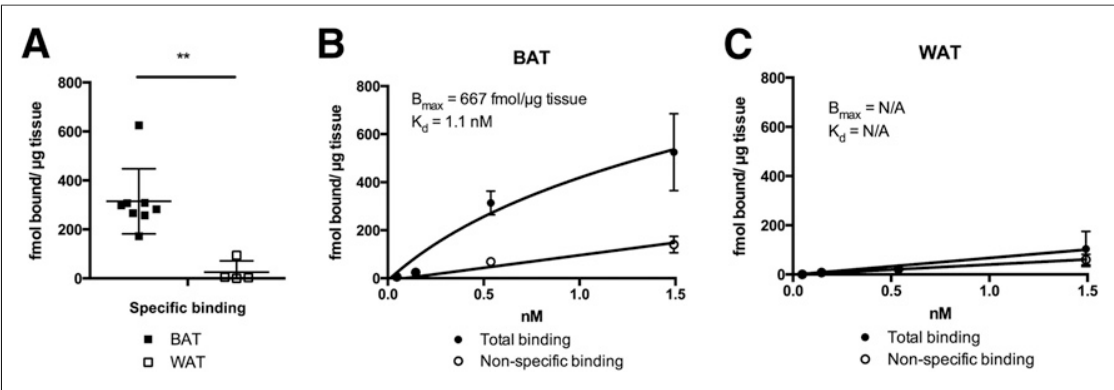

FIGURE 2. In vitro autoradiography of ${ }^{18} \mathrm{~F}-\mathrm{FMPEP}-\mathrm{d}_{2}$ on sections of BAT and WAT. (A) Displaceable CB1-specific binding was higher in BAT than in WAT at $0.5-1.5 \mathrm{nM}$ of added radiotracer $(P<$ 0.01). (B) Saturation of binding occurred at nanomolar concentrations of ${ }^{18} \mathrm{~F}-\mathrm{FMPEP}-\mathrm{d}_{2}$, with corresponding estimated receptor density of $667 \mathrm{fmol}$ of $\mathrm{CB} 1 \mathrm{~s}$ per microgram of BAT tissue. No appreciable binding was found in WAT, and neither dissociation constant $\left(\mathrm{K}_{\mathrm{d}}\right)$ nor receptor density $\left(\mathrm{B}_{\max }\right)$ could be estimated. ${ }^{* \star} P<0.01$.

\section{DISCUSSION}

Here, we have shown the feasibility of noninvasive assessment of the CB1 in deposits of BAT in a rodent model. The implications of this are several, both for development of novel approaches for activating BAT and for quantifying BAT mass (both native and induced) in vivo.

Systemically administration of CB1 antagonists, most notably rimonabant, has been shown to induce weight loss in humans. This effect can be attributed partly to induction of satiety by CB1 antagonism in the CNS (9). More recently it was also shown that peripheral CB1 antagonism increased energy expenditure in rodents, with subsequent weight 


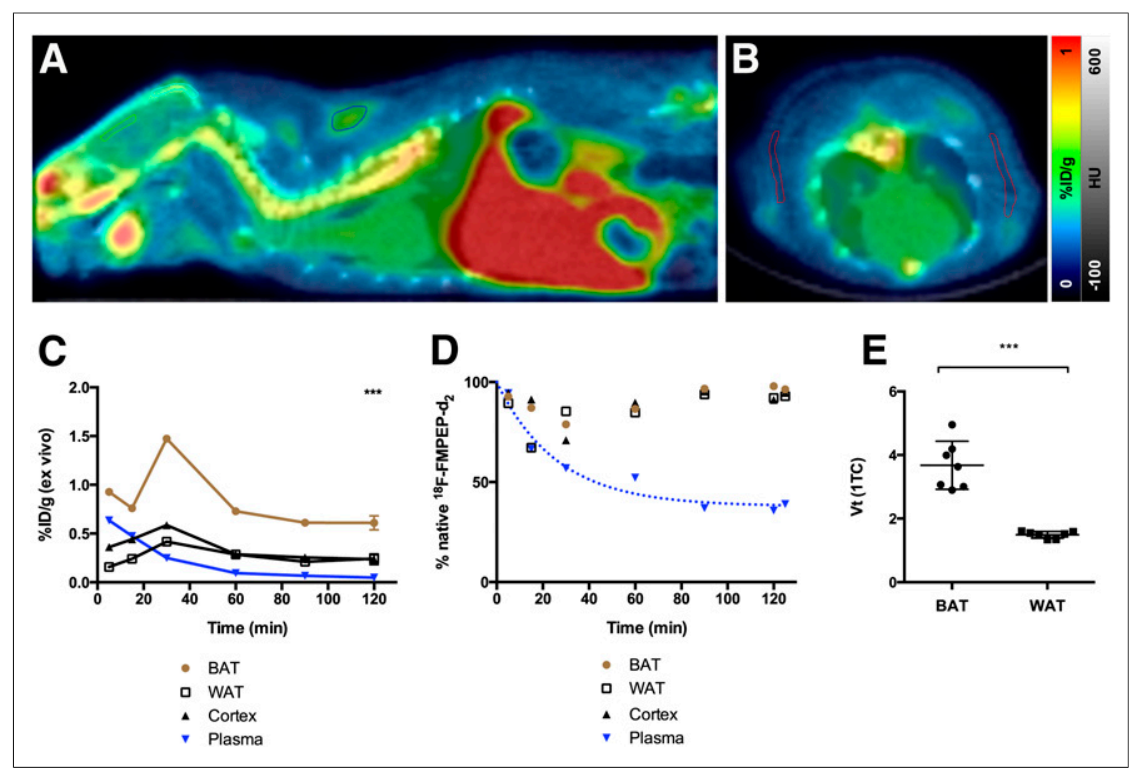
PET. Dark blue region indicates iBAT, green region indicates cerebral cortex, and light blue region indicates bone in skull. Red regions indicate subcutaneous WAT. (C) Ex vivo organ distribution shows retention in iBAT but less so in WAT and rapid clearance from blood plasma. Retention in iBAT is significantly higher than in WAT 120 min after ${ }^{18} \mathrm{~F}-\mathrm{FMPEP}-\mathrm{d}_{2}$ administration $(P<0.001)$. High uptake in bone is due to defluorination and subsequent ${ }^{18} \mathrm{~F}^{-}$binding into hydroxyapatitite in areas with bone formation. Metabolite analysis shows that retained tracer in BAT and other tissues consists of nearly $100 \%$ intact ${ }^{18}$ F-FMPEP- $d_{2}$. (D) Metabolism in blood plasma (at least partially from defluorination) followed a monoexponential model. 1TC model best described tracer kinetic uptake in WAT and could also describe uptake in BAT. (E) Vt was higher in iBAT than WAT. ${ }^{\star \star *} P<$ 0.001. PET images in $A$ and $B$ are summations of PET acquisition 0-35 min after administration of ${ }^{18} \mathrm{~F}-\mathrm{FMPEP}-\mathrm{d}_{2}$. PET color maps are normalized to 1 percentage injected dose per gram of tissue (\%ID/g), and CT color maps are normalized from -100 to 600 Hounsfield units (HUs).
FIGURE 3. (A and B) ${ }^{18} \mathrm{~F}-\mathrm{FMPEP}-\mathrm{d}_{2}$ accumulates at location of iBAT in rodents as measured by

been found that BAT is more prevalent in some populations, such as young, lean women, and rare in other groups, such as obese persons. These correlations are remarkable in themselves, but it is currently not known if lack of BAT is caused by long-standing obesity (e.g., some form of BAT exhaustion) or if the absence of endogenous BAT from an early age predisposes humans toward the development of obesity later in life.

Here, we proposed the $\mathrm{CB} 1$ as an imaging biomarker for the presence of BAT. Both immunohistochemistry and PET results presented here show that $\mathrm{CB} 1$ is colocalized with BAT or the exclusive BAT marker UCP-1 both in rodents and in humans. Importantly, subcutaneous WAT is almost devoid of CB1, suggesting that $\mathrm{CB} 1$ can be used as a surrogate marker for the UCP-1 and consequently BAT deposits. This is, to our knowledge, the first study to show extensive CB1 density in BAT. However, gene expression studies have reported CB1 expression across several tissues previously including BAT, at least in mice, in which CB1 was not overexpressed compared with WAT (13). The CB1 gene expression levels in human BAT are currently unknown.

The radioligand ${ }^{18}$ F-FMPEP- $\mathrm{d}_{2}$ can identify CB1-rich peripheral regions including BAT without the elaborated cold treatment required for the detection of BAT by ${ }^{18} \mathrm{~F}-$ FDG PET. Presently, it is unknown whether loss (10). The unwanted side effects of CB1 antagonism are exclusively centrally mediated and include severe ones such as suicidal ideation. Consequently, development of rimonabant for weight loss purposes was discontinued. Currently, novel CB1 antagonists with limited penetration of the blood-brain barrier (BBB) are being developed to exploit the peripherally mediated weight loss while avoiding the centrally mediated side effects.

We proposed that the methodology described in this study can be used to further understand the function of $\mathrm{CB} 1$ in BAT, including assessment of $\mathrm{CB} 1$ up- or downregulation during acute or chronic activation of BAT induced either by cold or by pharmaceutical intervention (e.g., adrenoreceptor agonism, irisin). Additionally, it is likely feasible to directly evaluate the receptor occupancy in BAT by novel non-BBB-penetrating CB1-targeted antagonists and the correlation between $\mathrm{CB} 1$ occupancy and BAT activation. Indeed, it was recently reported that intravenous pretreatment with the CB1 antagonist TM388837 induced a decreased binding of ${ }^{18} \mathrm{~F}-\mathrm{FMPEP}-\mathrm{d}_{2}$ in presumed BAT in nonhuman primates (11).

The presence of BAT in human adults is highly heterogeneous between individuals and difficult or impossible to predict without imaging methodologies. Currently, the only method for confirming BAT in individual humans is the examination of cold-induced glucose utilization in the supraclavicular regions by PET. Recently, it was also shown that BAT activated by $\beta 3$ adrenergic receptor agonism can be measured in a similar manner (12). With the use of these advanced methodologies, it has
CB1 is expressed in similar amounts in each brown adipocyte or if there are large differences. This must be further evaluated for understanding the potential of $\mathrm{CB} 1$ as a surrogate marker for BAT mass in vivo.

Other limitations of the current study include the translation from rodents to humans. ${ }^{18} \mathrm{~F}-\mathrm{FMPEP}-\mathrm{d}_{2}$ exhibits high defluorination with subsequent uptake in bone in rats. In humans, however, this is not seen in appreciable amounts. In fact, the reason for the deuteration of the tracer was intended to decrease the rate of defluorination.

Because of the marked difference in CB1 expression, ${ }^{18} \mathrm{~F}-\mathrm{FMPEP}-\mathrm{d}_{2}$ can potentially differentiate between BAT and WAT. White to brown fat conversion (browning) is a key step for exploring the potential use of BAT activation in obesity treatment. Only few reports on detection of browning in vivo have been reported, including the use of the norepinephrine transporter as a surrogate imaging biomarker due to the extensive sympathetic innervation of BAT (14-16). However, the activity and up- and downregulation of norepinephrine transporter and other components of the noradrenergic system are tightly connected to the activation of BAT. For example, induction of anesthesia completely blunts both the activation of BAT and the use and uptake of norepinephrine transporter by substrates such as ephedrine $(15,17)$. It is therefore likely that the ${ }^{11} \mathrm{C}$-hydroxyephedrine PET quantitation methodologies can underestimate the amount of BAT or browning deposits in WAT under several physiologic conditions. We therefore instead propose to further validate ${ }^{18} \mathrm{~F}-\mathrm{FMPEP}-\mathrm{d}_{2}$ as a robust PET marker for detection of induced browning. 


\section{CONCLUSION}

The CB1 is a surrogate biomarker for BAT and can be quantified by ${ }^{18} \mathrm{~F}-\mathrm{FMPEP}-\mathrm{d}_{2}$ PET. This novel noninvasive approach can be used to further elucidate the role of the central and peripheral endocannabinoid system in regulation and activation of BAT and energy expenditure. Additionally, it can be used for target engagement studies in the development of novel antiobesity CB1 antagonists.

\section{DISCLOSURE}

The costs of publication of this article were defrayed in part by the payment of page charges. Therefore, and solely to indicate this fact, this article is hereby marked "advertisement" in accordance with 18 USC section 1734. This study was conducted within the Centre of Excellence in Cardiovascular and Metabolic Reseach, supported by the Academy of Finland, the University of Turku, University of Eastern Finland, the Turku University Hospital, and Åbo Academy University. Additional financing was received from the DIABAT FP7, the Swedish Medical Council, and the Finnish Diabetes Foundation. No other potential conflict of interest relevant to this article was reported.

\section{ACKNOWLEDGMENTS}

We thank Aake Honkaniemi, Elisa Riuttala, Marko Vehmanen, Ola Åberg, and Veronika Aspund for excellent technical assistance.

\section{REFERENCES}

1. Virtanen KA, Lidell ME, Orava J, et al. Functional brown adipose tissue in healthy adults. $N$ Engl J Med. 2009;360:1518-1525.

2. Peirce V, Vidal-Puig A. Regulation of glucose homoeostasis by brown adipose tissue. Lancet Diabetes Endocrinol. 2013;1:353-360.

3. Bajzer M, Olivieri M, Haas MK, et al. Cannabinoid receptor 1 (CB1) antagonism enhances glucose utilisation and activates brown adipose tissue in diet-induced obese mice. Diabetologia. 2011;54:3121-3131.
4. Verty AN, Allen AM, Oldfield BJ. The effects of rimonabant on brown adipose tissue in rat: implications for energy expenditure. Obesity (Silver Spring). 2009;17: 254-261.

5. Boon MR, Kooijman S, van Dam AD, et al. Peripheral cannabinoid 1 receptor blockade activates brown adipose tissue and diminishes dyslipidemia and obesity. FASEB J. 2014;28:5361-5375.

6. Perwitz N, Wenzel J, Wagner I, et al. Cannabinoid type 1 receptor blockade induces transdifferentiation towards a brown fat phenotype in white adipocytes. Diabetes Obes Metab. 2010;12:158-166.

7. Terry GE, Hirvonen J, Liow JS, et al. Imaging and quantitation of cannabinoid CB1 receptors in human and monkey brains using ${ }^{18} \mathrm{~F}$-labeled inverse agonist radioligands. J Nucl Med. 2010;51:112-120.

8. Donohue SR, Krushinski JH, Pike VW, et al. Synthesis, ex vivo evaluation, and radiolabeling of potent 1,5-diphenylpyrrolidin-2-one cannabinoid subtype-1 receptor ligands as candidates for in vivo imaging. J Med Chem. 2008;51:58335842 .

9. Hodge J, Bow JP, Plyler KS, et al. The cannabinoid CB1 receptor inverse agonist AM 251 and antagonist AM 4113 produce similar effects on the behavioral satiety sequence in rats. Behav Brain Res. 2008;193:298-305.

10. Kunz I, Meier MK, Bourson A, Fisseha M, Schilling W. Effects of rimonabant, a cannabinoid CB1 receptor ligand, on energy expenditure in lean rats. Int J Obes (Lond). 2008;32:863-870.

11. Takano A, Gulyás B, Varnäs K, et al. Low brain CB1 receptor occupancy by a second generation CB1 receptor antagonist TM38837 in comparison with rimonabant in nonhuman primates: a PET study. Synapse. 2014;68:89-97.

12. Cypess AM, Weiner LS, Roberts-Toler C, et al. Activation of human brown adipose tissue by a $\beta 3$-adrenergic receptor agonist. Cell Metab. 2015;21:33-38.

13. Cnr1 (cannabinoid receptor 1 (brain)). BioGPS website. http://biogps.org/ \#goto=genereport\&id=12801. Updated November 19, 2012. Accessed September $10,2015$.

14. Thackeray JT, Beanlands RS, Dasilva JN. Presence of specific ${ }^{11} \mathrm{C}$-meta-hydroxyephedrine retention in heart, lung, pancreas, and brown adipose tissue. $\mathrm{J} \mathrm{Nucl}$ Med. 2007;48:1733-1740.

15. Quarta C, Lodi F, Mazza R, et al. ${ }^{11} \mathrm{C}$-meta-hydroxyephedrine PET/CT imaging allows in vivo study of adaptive thermogenesis and white-to-brown fat conversion. Mol Metab. 2013;2:153-160.

16. Lin SF, Fan X, Yeckel CW, et al. Ex vivo and in vivo evaluation of the norepinephrine transporter ligand $\left[{ }^{11} \mathrm{C}\right] \mathrm{MRB}$ for brown adipose tissue imaging. $\mathrm{Nucl}$ Med Biol. 2012;39:1081-1086.

17. Ohlson KB, Mohell N, Cannon B, Lindahl SG, Nedergaard J. Thermogenesis in brown adipocytes is inhibited by volatile anesthetic agents: a factor contributing to hypothermia in infants? Anesthesiology. 1994;81:176-183. 